CARPATHIAN J. MATH.

Volume 37 (2021), No. 1,

Pages 81 - 89
Online version at https : //www. carpathian. cunbm. utcluj. ro/

Print Edition: ISSN 1584 - 2851; Online Edition: ISSN 1843 - 4401

DOI: https://doi.org/10.37193/CJM.2021.01.08

\title{
A new complex generalized Bernstein-Schurer operator
}

\author{
NURSEL ÇETIN
}

ABSTRACT. In this paper, we consider the complex form of a new generalization of Bernstein-Schurer operators. We obtain some quantitative upper estimates for the approximation of these operators attached to analytic functions. Moreover, we prove that these operators preserve some properties of the original function such as univalence, starlikeness, convexity and spirallikeness.

\section{INTRODUCTION}

In 1912, the classical Bernstein polynomials

$$
B_{n}(f, x)=\sum_{k=0}^{n} f\left(\frac{k}{n}\right)\left(\begin{array}{l}
n \\
k
\end{array}\right) x^{k}(1-x)^{n-k}, \quad x \in[0,1]
$$

for any $n \in \mathbb{N}$ and $f \in C[0,1]$, the space of all real valued continuous functions on $[0,1]$, were proposed by Bernstein [2] as one of the simplest way to prove Weierstrass Approximation Theorem. Then, considering many advantages in terms of their elegant structure, simplicity and useful approximation properties, a large number of researchers have studied intensively the discovery of their various generalizations and modifications in different ways.

In 1962, considering a given non-negative integer $p$, Schurer [9] introduced and studied the following generalization of Bernstein operators $B_{n, p}: C[0,1+p] \rightarrow C[0,1]$ defined as

$$
B_{n, p}(f, x)=\sum_{k=0}^{n+p} f\left(\frac{k}{n}\right)\left(\begin{array}{c}
n+p \\
k
\end{array}\right) x^{k}(1-x)^{n+p-k}, x \in[0,1]
$$

for any $n \in \mathbb{N}$ and $f \in C[0,1+p]$. The special case $p=0$ gives the classical Bernstein polynomials.

In 1981, by using a probabilistic method, Stancu [10] constructed a new family of linear positive operators which is called as Stancu operators, is given by

$$
L_{n, r}(f ; x)=\sum_{k=0}^{n-r} p_{n-r, k}(x)\left[(1-x) f\left(\frac{k}{n}\right)+x f\left(\frac{k+r}{n}\right)\right],
$$

where $f \in C[0,1], r$ is a non-negative integer, $n \in \mathbb{N}$ such that $n>2 r, x \in[0,1]$ and $p_{n, k}(x)=\left(\begin{array}{l}n \\ k\end{array}\right) x^{k}(1-x)^{n-k}$ and investigated some approximation properties of these operators. Note that for the special cases $r=0$ and $r=1$ they reduce to the Bernstein polynomials. In [3], Bustamante and Quesada obtained an asymptotic property related to Voronovskaja type theorem for the Stancu operators $L_{n, r}$. In [12], Yang et al. studied multivariate Stancu operators on a simplex. The authors gave a symmetric property and proved that Lipschitz property of the original function is preserved by the multivariate Stancu operators.

Received: 30.11.2018. In revised form: 15.07.2020. Accepted: 22.07.2020

2010 Mathematics Subject Classification. 30E10, 41A25, 41A28, 30C45.

Key words and phrases. Complex Stancu operator, complex Bernstein-Schurer operator, quantitative estimate, simultaneous approximation, univalence, starlikeness, convexity, spirallikeness. 
Recently, Gal [6] presented the overconvergence properties of complex Bernstein polynomials. In [6], the author obtained the order of simultaneous approximation and a Voronovskaja-type theorem with a quantitative estimate for these operators on compact disks. Also, Gal compiled similar results for the well-known complex operators in his book [6]. Later on, the problem of approximation of complex operators has attracted the attention of many researchers. Recently, Jiang et al. [8] introduced a complex form of Stancu type Bernstein-Schurer polynomials and their Kantorovich variants and obtained: quantitative upper estimates for simultaneous approximation, quantitative Voronovskajatype results and the exact order of approximation by these operators attached to analytic functions in closed disks. In this paper, inspired by the real case in [10] and the above works concerning the operators in complex domains, we introduce the new complex generalized Bernstein-Schurer operators defined as follows

$$
L_{n, p}^{r}(f ; z)=\sum_{k=0}^{n+p-r}\left(\begin{array}{c}
n+p-r \\
k
\end{array}\right) z^{k}(1-z)^{n+p-r-k}\left[(1-z) f\left(\frac{k}{n}\right)+z f\left(\frac{k+r}{n}\right)\right]
$$

where $r$ is a non-negative integer, $n \in \mathbb{N}$ such that $n>2 r, p \in \mathbb{N} \cup\{0\}, z \in \mathbb{C}$ and $f$ is a complex-valued analytic function in an open disk with $R>1$ and centered at the origin. We call the new operators $L_{n, p}^{r}$ as complex Stancu-Schurer operators. Note that when $r=0$ or $r=1$ with $p=0$, these operators reduce to the classical complex Bernstein polynomials $B_{n}(f ; z)=L_{n, 0}^{0}(f ; z)=L_{n, 0}^{1}(f ; z)$. For $r=0$ or $r=1$, the operators $L_{n, p}^{r}$ give the complex Bernstein-Schurer operators $B_{n, p}(f ; z)=L_{n, p}^{0}(f ; z)=L_{n, p}^{1}(f ; z)$. Also, in the case of $p=0$, these operators reduce to complex Stancu operators studied in [4]. Here, we obtain a quantitative upper estimate for the complex Stancu-Schurer operators given in (1.4) and also investigate some geometric properties of these operators attached to analytic functions.

\section{APPROXIMATION BY COMPLEX STANCU-SCHURER OPERATORS}

Throughout the paper, we use the indication $e_{k}(z):=z^{k}, k \in \mathbb{N} \cup\{0\}, z \in \mathbb{C}$, $D_{R}=\{z \in \mathbb{C}:|z|<R, R>1\}, \overline{D_{\rho}}=\{z \in \mathbb{C}:|z| \leq \rho, 1 \leq \rho<R\}$ and $\|f\|_{\rho}=\max \{|f(z)|:|z| \leq \rho\}$. Let $\Delta_{h}^{k}$ denote the finite difference of order $k$ with step $h$, that is

$$
\Delta_{h}^{k} f(x)=k ! h^{k}[x, x+h, \cdots, x+k h ; f]
$$

(see p. 121 of [5]). By means of finite differences, we can easily deduce the following representation as in the case of Stancu operators $L_{n, r}$ given by the formula (1.7) in [11]. This formula holds in complex setting too.

Lemma 2.1. The operators $L_{n, p}^{r}(f ; z)$ defined by (1.4) have the representation formula

$$
L_{n, p}^{r}(f ; z)=\sum_{k=0}^{n+p-r}\left(\begin{array}{c}
n+p-r \\
k
\end{array}\right)\left[(1-z) \Delta_{1 / n}^{k} f(0)+z \Delta_{1 / n}^{k} f\left(\frac{r}{n}\right)\right] z^{k},
$$

where $\Delta_{1 / n}^{k}$ is given by (2.5) with $h=\frac{1}{n}$.

Lemma 2.2. Suppose that $r$ is a non-negative integer, $n \in \mathbb{N}$ such that $n>2 r, p, k \in \mathbb{N} \cup\{0\}$ and $z \in \mathbb{C}$. Then, we have

$$
\begin{aligned}
L_{n, p}^{r}\left(e_{k+1} ; z\right)= & \frac{z(1-z)}{n}\left(L_{n, p}^{r}\left(e_{k} ; z\right)\right)^{\prime}+\left[z+\frac{(r-1)(1-z)+p z}{n}\right] L_{n, p}^{r}\left(e_{k} ; z\right) \\
& +\frac{(1-r)(1-z)}{n}\left(\frac{n-r}{n}\right)^{k} B_{n-r, p}\left(e_{k} ; z\right),
\end{aligned}
$$


where $B_{n, p}$ is the complex Bernstein-Schurer operator.

Proof. From (1.4), we can write

$$
L_{n, p}^{r}\left(e_{k} ; z\right)=\sum_{j=0}^{n+p-r}\left(\begin{array}{c}
n+p-r \\
j
\end{array}\right) z^{j}(1-z)^{n+p-r-j}\left[(1-z)\left(\frac{j}{n}\right)^{k}+z\left(\frac{j+r}{n}\right)^{k}\right] .
$$

Differentiating $L_{n, p}^{r}\left(e_{k} ; z\right)$ with respect to $z \neq 0$, by some calculations we have

$$
\begin{aligned}
& \left(L_{n, p}^{r}\left(e_{k} ; z\right)\right)^{\prime} \\
& =\sum_{j=0}^{n+p-r}\left(\begin{array}{c}
n+p-r \\
j
\end{array}\right)\left[j z^{j-1}(1-z)^{n+p-r-j}-(n+p-r-j) z^{j}(1-z)^{n+p-r-j-1}\right] \times \\
& \left.(1-z)\left(\frac{j}{n}\right)^{k}+z\left(\frac{j+r}{n}\right)^{k}\right]+\sum_{j=0}^{n+p-r}\left(\begin{array}{c}
n+p-r \\
j
\end{array}\right) z^{j}(1-z)^{n+p-r-j}\left[\left(\frac{j+r}{n}\right)^{k}-\left(\frac{j}{n}\right)^{k}\right] \\
& =\frac{1}{z(1-z)} \sum_{j=0}^{n+p-r}\left(\begin{array}{c}
n+p-r \\
j
\end{array}\right) z^{j}(1-z)^{n+p-r-j} j\left[(1-z)\left(\frac{j}{n}\right)^{k}\right]+\frac{1}{z(1-z)} \sum_{j=0}^{n+p-r}\left(\begin{array}{c}
n+p-r \\
j
\end{array}\right) \times \\
& z^{j}(1-z)^{n+p-r-j} j\left[z\left(\frac{j+r}{n}\right)^{k}\right]-\frac{(n+p-r)^{n+p-r}}{(1-z)} \sum_{j=0}^{n+p-r}\left(\begin{array}{c}
j \\
j
\end{array}\right) z^{j}(1-z)^{n+p-r-j} \times z^{j}(1-z)^{n+p-r-j}\left[\left(\frac{j+r}{n}\right)^{k}-\left(\frac{j}{n}\right)^{k}\right] \\
& {\left[(1-z)\left(\frac{j}{n}\right)^{k}+z\left(\frac{j+r}{n}\right)^{k}\right]+\sum_{j=0}^{n+p-r}\left(\begin{array}{c}
n+p-r \\
j
\end{array}\right)}
\end{aligned}
$$

Considering that $j$ can be written as $j+r-r$, we get

$$
\begin{aligned}
& \left(L_{n, p}^{r}\left(e_{k} ; z\right)\right)^{\prime}=\frac{n}{z(1-z)} \sum_{j=0}^{n+p-r}\left(\begin{array}{c}
n+p-r \\
j
\end{array}\right) z^{j}(1-z)^{n+p-r-j}\left[(1-z)\left(\frac{j}{n}\right)^{k+1}\right]+\frac{n}{z(1-z)} \times \\
& \sum_{j=0}^{n+p-r}\left(\begin{array}{c}
n+p-r \\
j
\end{array}\right) z^{j}(1-z)^{n+p-r-j}\left[z\left(\frac{j+r}{n}\right)^{k+1}\right]-\frac{r}{z(1-z)} \sum_{j=0}^{n+p-r}\left(\begin{array}{c}
n+p-r \\
j
\end{array}\right) z^{j} \times \\
& (1-z)^{n+p-r-j}\left[z\left(\frac{j+r}{n}\right)^{k}\right]-\frac{(n+p-r)}{(1-z)} \sum_{j=0}^{n+p-r}\left(\begin{array}{c}
n+p-r \\
j
\end{array}\right) z^{j}(1-z)^{n+p-r-j} \times \\
& {\left[(1-z)\left(\frac{j}{n}\right)^{k}+z\left(\frac{j+r}{n}\right)^{k}\right]+\frac{1}{z} \sum_{j=0}^{n+p-r}\left(\begin{array}{c}
n+p-r \\
j
\end{array}\right) z^{j}(1-z)^{n+p-r-j}\left[z\left(\frac{j+r}{n}\right)^{k}\right]} \\
& -\sum_{j=0}^{n+p-r}\left(\begin{array}{c}
n+p-r \\
j
\end{array}\right) z^{j}(1-z)^{n+p-r-j}\left(\frac{j}{n}\right)^{k}
\end{aligned}
$$




$$
\begin{aligned}
& =\frac{n}{z(1-z)} \sum_{j=0}^{n+p-r}\left(\begin{array}{c}
n+p-r \\
j
\end{array}\right) z^{j}(1-z)^{n+p-r-j}\left[(1-z)\left(\frac{j}{n}\right)^{k+1}+z\left(\frac{j+r}{n}\right)^{k+1}\right]+\frac{(1-z-r)}{z(1-z)} \times \\
& \sum_{j=0}^{n+p-r}\left(\begin{array}{c}
n+p-r \\
j
\end{array}\right) z^{j}(1-z)^{n+p-r-j}\left[z\left(\frac{j+r}{n}\right)^{k}\right]-\frac{(n+p-r)}{(1-z)} \sum_{j=0}^{n+p-r}\left(\begin{array}{c}
n+p-r \\
j
\end{array}\right) z^{j}(1-z)^{n+p-r-j} \times \\
& {\left[(1-z)\left(\frac{j}{n}\right)^{k}+z\left(\frac{j+r}{n}\right)^{k}\right]-\sum_{j=0}^{n+p-r}\left(\begin{array}{c}
n+p-r \\
j
\end{array}\right) z^{j}(1-z)^{n+p-r-j}\left(\frac{j}{n}\right)^{k}} \\
& =\frac{n}{z(1-z)} L_{n, p}^{r}\left(e_{k+1} ; z\right)+\frac{(1-z-r)}{z(1-z)} L_{n, p}^{r}\left(e_{k} ; z\right)-\frac{(1-z-r)}{z(1-z)} \sum_{j=0}^{n+p-r}\left(\begin{array}{c}
n+p-r \\
j
\end{array}\right)(1-z)^{n+p-r-j} \times \\
& z^{j}\left[(1-z)\left(\frac{j}{n}\right)^{k}\right]-\frac{(n+p-r)}{(1-z)} L_{n, p}^{r}\left(e_{k} ; z\right)-\sum_{j=0}^{n+p-r}\left(\begin{array}{c}
n+p-r \\
j
\end{array}\right) z^{j}(1-z)^{n+p-r-j}\left(\frac{j}{n}\right)^{k} \\
& =\frac{n}{z(1-z)} L_{n, p}^{r}\left(e_{k+1} ; z\right)-\frac{[(n+p-r) z-1+z+r]}{z(1-z)} L_{n, p}^{r}\left(e_{k} ; z\right)+\left(\frac{r-1}{z}\right) \sum_{j=0}^{n+p-r}\left(\begin{array}{c}
n+p-r \\
j
\end{array}\right) \times \\
& z^{j}(1-z)^{n+p-r-j}\left(\frac{j}{n}\right)^{k} \\
& =\frac{n}{z(1-z)} L_{n, p}^{r}\left(e_{k+1} ; z\right)-\frac{[(n+p-r) z+r+z-1]}{z(1-z)} L_{n, p}^{r}\left(e_{k} ; z\right)+\left(\frac{r-1}{z}\right)\left(\frac{n-r}{n}\right)^{k} B_{n-r, p}\left(e_{k} ; z\right),
\end{aligned}
$$

which gives the desired statement.

Theorem 2.1. Suppose that $r$ is a non-negative integer, $n \in \mathbb{N}$ such that $n>2 r$ and $f: D_{R} \rightarrow \mathbb{C}$ is analytic in $D_{R}$, for fixed $p \in \mathbb{N} \cup\{0\}$ and $R>p+1$ with $f(z)=\sum_{k=0}^{\infty} c_{k} z^{k}$.

(i) Let $1 \leq \rho$ and $\rho(p+1)<R$ be arbitrary fixed. For all $|z| \leq \rho$ and $n \in \mathbb{N}$, we have

$$
\left|L_{n, p}^{r}(f ; z)-f(z)\right| \leq M_{\rho, n, p}^{r}(f),
$$

where

$$
\begin{aligned}
0< & M_{\rho, n, p}^{r}(f)=\sum_{k=1}^{\infty}\left|c_{k}\right|\left\{(3 \rho+3+r)(1+\rho) \frac{k(k+1)}{2} \frac{[(p+1) \rho]^{k-1}}{n}\right. \\
& \left.+(3 \rho+2)[r(1+\rho)+p \rho] k \frac{[(p+1) \rho]^{k-1}}{n}+4 k(k-1) \frac{[(p+1) \rho]^{k}}{n+p-r}\right\}<\infty .
\end{aligned}
$$

(ii) Also, if $1 \leq \rho<\rho_{1} \leq \rho_{1}(p+1)<R$, then for all $|z| \leq \rho$ and $n, j \in \mathbb{N}$, we have

$$
\left|\left(L_{n, p}^{r}(f ; z)\right)^{(j)}-f^{(j)}(z)\right| \leq \frac{M_{\rho_{1, n, p}}^{r}(f) j ! \rho_{1}}{\left(\rho_{1}-\rho\right)^{j+1}},
$$

where $M_{\rho_{1, n, p}}^{r}(f)$ is given as in $(i)$.

Proof. As in the case of Bernstein polynomials (see, p. 9 in [6]), we easily obtain $L_{n, p}^{r}(f ; z)=$ $\sum_{k=0}^{\infty} c_{k} L_{n, p}^{r}\left(e_{k} ; z\right)$, which follows

$$
\left|L_{n, p}^{r}(f ; z)-f(z)\right| \leq \sum_{k=0}^{\infty}\left|c_{k}\right|\left|L_{n, p}^{r}\left(e_{k} ; z\right)-e_{k}(z)\right|
$$


In order to estimate $\left|L_{n, p}^{r}\left(e_{k} ; z\right)-e_{k}(z)\right|$ for fixed $n \in \mathbb{N}$, we consider the cases : $0 \leq k \leq$ $n+p-r$ and $k>n+p-r$.

Case 1: For $k=0$, we have $L_{n, p}^{r}\left(e_{k} ; z\right)-e_{k}(z)=0$. So, we consider the case $1 \leq k \leq$ $n+p-r$. Denoting $\pi_{n, p, k}^{r}(z)=L_{n, p}^{r}\left(e_{k} ; z\right)$, by the recurrence formula in (2.7) we get

$$
\begin{array}{ll}
\pi_{n, p, k}^{r}(z)- & e_{k}(z)=\frac{z(1-z)}{n}\left[\pi_{n, p, k-1}^{r}(z)-z^{k-1}\right]^{\prime}+\left[z+\frac{(r-1)(1-z)+p z}{n}\right] \times \\
& {\left[\pi_{n, p, k-1}^{r}(z)-z^{k-1}\right]+\frac{(1-r)(1-z)}{n}\left(\frac{n-r}{n}\right)^{k-1}\left[B_{n-r, p}\left(e_{k-1} ; z\right)-z^{k-1}\right]} \\
& +\frac{[(k+r-2)(1-z)+p z]}{n} z^{k-1}+\frac{(1-r)(1-z)}{n}\left(\frac{n-r}{n}\right)^{k-1} z^{k-1} .
\end{array}
$$

Using the Bernstein's inequality (see p. 3 of [6]) in the above recurrence, by some calculations, for $|z| \leq \rho, \rho \geq 1$, we have

$$
\begin{aligned}
& \left|\pi_{n, p, k}^{r}(z)-e_{k}(z)\right| \leq \frac{\rho(1+\rho)}{n} \frac{(k-1)}{\rho}\left\|\pi_{n, p, k-1}^{r}-e_{k-1}\right\|_{\rho}+\left[\rho+\frac{(1+r)(1+\rho)+p \rho}{n}\right] \times \\
& \left|\pi_{n, p, k-1}^{r}(z)-e_{k-1}(z)\right|+\frac{(1+r)(1+\rho)}{n}\left(\frac{n-r}{n}\right)^{k-1}\left|B_{n-r, p}\left(e_{k-1} ; z\right)-e_{k-1}(z)\right| \\
& \quad+\frac{[(k+r)(1+\rho)+p \rho]}{n} \rho^{k-1}+\frac{(1+r)(1+\rho)}{n}\left(\frac{n-r}{n}\right)^{k-1} \rho^{k-1} \\
& \leq \\
& \quad \frac{[(k+r)(1+\rho)+p \rho]}{n}\left\|\pi_{n, p, k-1}^{r}-e_{k-1}\right\|_{\rho}+\rho\left|\pi_{n, p, k-1}^{r}(z)-e_{k-1}(z)\right| \\
& \quad+\frac{(1+r)(1+\rho)}{n}\left|B_{n-r, p}\left(e_{k-1} ; z\right)-e_{k-1}(z)\right|+\frac{[(k+r)(1+\rho)+p \rho]}{n} \rho^{k-1}+\frac{(1+r)(1+\rho)}{n} \rho^{k-1} \\
& \leq \rho\left|\pi_{n, p, k-1}^{r}(z)-e_{k-1}(z)\right|+\frac{[(k+r)(1+\rho)+p \rho]}{n}\left[\left\|\pi_{n, p, k-1}^{r}\right\|_{\rho}+\left\|e_{k-1}\right\|_{\rho}\right] \\
& \quad+\frac{(1+r)(1+\rho)}{n}\left|B_{n-r, p}\left(e_{k-1} ; z\right)-e_{k-1}(z)\right|+\frac{[(k+r)(1+\rho)+p \rho]}{n} \rho^{k-1}+\frac{(1+r)(1+\rho)}{n} \rho^{k-1} .
\end{aligned}
$$

According to the estimates obtained in the proof of Theorem 2.1, p. 736 in [1], the last inequality follow s that

$$
\begin{gathered}
\left|\pi_{n, p, k}^{r}(z)-e_{k}(z)\right| \leq \rho\left|\pi_{n, p, k-1}^{r}(z)-e_{k-1}(z)\right|+\frac{[(k+r)(1+\rho)+p \rho]}{n} \times \\
{\left[\left\|\pi_{n, p, k-1}^{r}\right\|_{\rho}+\left\|e_{k-1}\right\|_{\rho}\right]+\frac{(1+r)(1+\rho)}{n}\left\{\frac{(k-1)(k-2)}{n-r+p}[(p+1) \rho]^{k-1}\right.} \\
\left.+\frac{1}{n-r}[(p+1) \rho]^{k-1}-\frac{\rho^{k-1}}{n-r}\right\}+\frac{[(k+r)(1+\rho)+p \rho]}{n} \rho^{k-1}+\frac{(1+r)(1+\rho)}{n} \rho^{k-1} \\
\leq \rho\left|\pi_{n, p, k-1}^{r}(z)-e_{k-1}(z)\right|+\frac{[(k+r)(1+\rho)+p \rho]}{n}\left\|\pi_{n, p, k-1}^{r}\right\|_{\rho}+\frac{(1+r)(1+\rho)}{n}\{(k-1) \times \\
\left.[(p+1) \rho]^{k-1}+[(p+1) \rho]^{k-1}-\rho^{k-1}\right\}+\frac{2[(k+r)(1+\rho)+p \rho]}{n} \rho^{k-1}+\frac{(1+r)(1+\rho)}{n} \rho^{k-1} \\
=\rho\left|\pi_{n, p, k-1}^{r}(z)-e_{k-1}(z)\right|+\frac{[(k+r)(1+\rho)+p \rho]}{n}\left\|\pi_{n, p, k-1}^{r}\right\|_{\rho}+\frac{2[(k+r)(1+\rho)+p \rho]}{n} \rho^{k-1} \\
\text { (2.9) }
\end{gathered}
$$


Now, let us obtain an upper estimate for $\left\|\pi_{n, p, k-1}^{r}\right\|_{\rho}$. For this aim, using the operator's representation via divided difference in (2.6), by some arrangements we get

$$
\begin{aligned}
& \left|\pi_{n, p, k}^{r}(z)\right| \leq \sum_{j=0}^{k}\left(\begin{array}{c}
n+p-r \\
j
\end{array}\right) \frac{j !}{n^{j}}\left\{(1+\rho)\left[0, \ldots, \frac{j}{n} ; e_{k}\right]+\rho\left[\frac{r}{n}, \frac{r+1}{n}, \ldots, \frac{r+j}{n} ; e_{k}\right]\right\} \rho^{j} \\
& \leq \rho^{k}\left\{(1+\rho) \sum_{j=0}^{n+p}\left(\begin{array}{c}
n+p \\
j
\end{array}\right) \frac{j !}{n^{j}}\left[0, \ldots, \frac{j}{n} ; e_{k}\right]+\rho \sum_{j=0}^{n+p-r}\left(\begin{array}{c}
n+p-r \\
j
\end{array}\right) \frac{j !}{n^{j}}\left[\frac{r}{n}, \ldots, \frac{r+j}{n} ; e_{k}\right]\right\} \\
& =\rho^{k}\left\{(1+\rho) B_{n, p}\left(e_{k} ; 1\right)+\rho L_{n, p}^{r}\left(e_{k} ; 1\right)\right\}
\end{aligned}
$$

for $|z| \leq \rho$ with $1 \leq \rho<R$. Since $B_{n, p}(f ; 1)=f\left(\frac{n+p}{n}\right)$ for the Bernstein-Schurer operators and $L_{n, p}^{r}(f ; 1)=f\left(\frac{n+p}{n}\right)$ for the new operator, $(2.10)$ reduces to

$$
\begin{aligned}
\left|\pi_{n, p, k}^{r}(z)\right| & \leq \rho^{k}\left\{(1+\rho)\left(\frac{n+p}{n}\right)^{k}+\rho\left(\frac{n+p}{n}\right)^{k}\right\} \\
& \leq \rho^{k}(1+2 \rho)\left(1+\frac{p}{n}\right)^{k} \leq 3 \rho^{k+1}(1+p)^{k} \leq 3 \rho[(1+p) \rho]^{k} .
\end{aligned}
$$

Substituting the last inequality into (2.9), the followings can be easily obtained

$$
\begin{gathered}
\left|\pi_{n, p, k}^{r}(z)-e_{k}(z)\right| \leq \rho\left|\pi_{n, p, k-1}^{r}(z)-e_{k-1}(z)\right|+\frac{[(k+r)(1+\rho)+p \rho]}{n} 3 \rho[(p+1) \rho]^{k-1} \\
+\frac{2[(k+r)(1+\rho)+p \rho]}{n} \rho^{k-1}+\frac{k(1+r)(1+\rho)}{n}[(p+1) \rho]^{k-1} \\
\leq \rho\left|\pi_{n, p, k-1}^{r}(z)-e_{k-1}(z)\right|+\{(3 \rho+2)[(k+r)(1+\rho)+p \rho]+k(1+r)(1+\rho)\} \frac{[(p+1) \rho]^{k-1}}{n} \\
\leq[(p+1) \rho]\left|\pi_{n, p, k-1}^{r}(z)-e_{k-1}(z)\right|+\{(3 \rho+3+r)(1+\rho) k+(3 \rho+2)[r(1+\rho)+p \rho]\} \frac{[(p+1) \rho]^{k-1}}{n} .
\end{gathered}
$$

By writing $k=1,2, \ldots$ in the last inequality, step by step, one has

$$
\begin{aligned}
& \left|\pi_{n, p, k}^{r}(z)-e_{k}(z)\right| \leq \frac{[(p+1) \rho]^{k-1}}{n} \sum_{j=1}^{k}[(3 \rho+3+r)(1+\rho) j+(3 \rho+2)[r(1+\rho)+p \rho]] \\
\leq & \frac{[(p+1) \rho]^{k-1}}{n}\left[(3 \rho+3+r)(1+\rho) \frac{k(k+1)}{2}+(3 \rho+2)[r(1+\rho)+p \rho] k\right] .
\end{aligned}
$$

Case 2 : For $k>n+p-r \geq 1$ and $|z| \leq \rho$ with $1 \leq \rho \leq \rho(p+1)<R$, from (2.6) we have

$$
\begin{aligned}
& \left|\pi_{n, p, k}^{r}(z)-e_{k}(z)\right| \leq\left|\pi_{n, p, k}^{r}(z)\right|+\left|e_{k}(z)\right| \\
& \leq \sum_{j=0}^{n+p-r}\left(\begin{array}{c}
n+p-r \\
j
\end{array}\right) \frac{j !}{n^{j}}\left\{(1+\rho)\left[0, \frac{1}{n}, \ldots, \frac{j}{n} ; e_{k}\right]+\rho\left[\frac{r}{n}, \ldots, \frac{r+j}{n} ; e_{k}\right]\right\} \rho^{j}+\rho^{k} \\
& \leq \rho^{n+p-r}\left\{(1+\rho) \sum_{j=0}^{n+p}\left(\begin{array}{c}
n+p \\
j
\end{array}\right) \frac{j !}{n^{j}}\left[0, \frac{1}{n}, \ldots, \frac{j}{n} ; e_{k}\right]+\rho \sum_{j=0}^{n+p-r}\left(\begin{array}{c}
n+p-r \\
j
\end{array}\right) \frac{j !}{n^{j}}\left[\frac{r}{n}, \ldots, \frac{r+j}{n} ; e_{k}\right]\right\}+\rho^{k} \\
= & \rho^{n+p-r}\left\{(1+\rho) B_{n, p}\left(e_{k} ; 1\right)+\rho L_{n, p}^{r}\left(e_{k} ; 1\right)\right\}+\rho^{k} .
\end{aligned}
$$


Using the fact $B_{n, p}(f ; 1)=f\left(\frac{n+p}{n}\right)$ for the Bernstein-Schurer operators and $L_{n, p}^{r}(f ; 1)=f\left(\frac{n+p}{n}\right)$ for the new operator, the last inequality gives

$$
\begin{aligned}
\left|\pi_{n, p, k}^{r}(z)-e_{k}(z)\right| & \leq \rho^{n+p-r}\left\{(1+\rho)\left(\frac{n+p}{n}\right)^{k}+\rho\left(\frac{n+p}{n}\right)^{k}\right\}+\rho^{k} \\
& \leq 3 \rho^{n+p-r+1}\left(1+\frac{p}{n}\right)^{k}+\rho^{k} \leq 3 \rho^{n+p-r+1}(1+p)^{k}+\rho^{k} \\
& \leq 3 \rho^{k}(1+p)^{k}+\rho^{k} \leq 4[(p+1) \rho]^{k} \leq 4(n+p-r)[(p+1) \rho]^{k} \\
& \leq 4(k-1) \frac{k}{n+p-r}[(p+1) \rho]^{k}=\frac{4 k(k-1)}{n+p-r}[(p+1) \rho]^{k} .
\end{aligned}
$$

As a consequence, combining Case 2 with the above Case 1, we arrive at the desired inequality.

(ii) It can be easily obtained by the method used for the Bernstein operator (see p.8 in [6]). So we omit the details.

\section{SHAPE PRESERVING PROPERTIES OF THE COMPLEX STANCU-SCHURER OPERATORS}

In this section, we prove that beginning with an index, the complex Stancu-Schurer operators $L_{n, p}^{r}(f ; z)$ preserve some geometric properties such as starlikeness, convexity and spirallikeness in the unit disk.

Theorem 3.2. Suppose that $G \subset \mathbb{C}$ is open such that $\overline{D_{1}} \subset G$ and $f: G \rightarrow \mathbb{C}$ is analytic in $G$. Also, let $\gamma \in\left(-\frac{\pi}{2}, \frac{\pi}{2}\right)$.

(i) If $f$ is univalent in $\overline{D_{1}}$, then there exists an index $n_{0}$ depending on $f$ such that for all $n \geq n_{0}$, the complex Stancu-Schurer operators $L_{n, p}^{r}(f ; z)$ are univalent in $\overline{D_{1}}$.

(ii) If $f(0)=f^{\prime}(0)-1=0$ and $f$ is starlike (convex, spirallike of type $\gamma$, respectively) in $\overline{D_{1}}$, that is for all $z \in \overline{D_{1}}$

$$
\operatorname{Re}\left(\frac{z f^{\prime}(z)}{f(z)}\right)>0\left(\operatorname{Re}\left(\frac{z f^{\prime \prime}(z)}{f^{\prime}(z)}\right)+1>0, \operatorname{Re}\left(e^{i \gamma} \frac{z f^{\prime}(z)}{f(z)}\right)>0, \text { resp. }\right),
$$

then there exists an index $n_{0}$ depending on $f$ (and on $\gamma$ for spirallikeness) such that for all $n \geq$ $n_{0}$, the complex Stancu-Schurer operators $L_{n, p}^{r}(f ; z)$ are starlike (convex, spirallike of type $\gamma$, respectively) in $\overline{D_{1}}$.

Proof. $i$ ) If $f$ is univalent in $\overline{D_{1}}$, from the uniform convergence in Theorem 2.1 and the result concerning sequences of analytic functions converging locally uniformly to a univalent function, it is immediate that for sufficiently large $n$, the complex Stancu-Schurer operators $L_{n, p}^{r}(f ; z)$ are univalent in $\overline{D_{1}}$ (see, e.g., p. 194 Theorem 6.1.18 in [7]).

(ii) Firstly, suppose that $f(0)=f^{\prime}(0)-1=0$ and $f$ is starlike in $\overline{D_{1}}$. By Theorem $2.1(i i)$, we get that $L_{n, p}^{r}(f ; z) \rightarrow f(z),\left(L_{n, p}^{r}(f ; z)\right)^{\prime} \rightarrow f^{\prime}(z)$ and $\left(L_{n, p}^{r}(f ; z)\right)^{\prime \prime} \rightarrow f^{\prime \prime}(z)$ as $n \rightarrow$ $\infty$, uniformly in $\overline{D_{1}}$. Now, set $P_{n, p}^{r}(f ; z)=\frac{L_{n, p}^{r}(f ; z)}{(n+p-r) f\left(\frac{1}{n}\right)+f\left(\frac{r}{n}\right)}$, well defined for sufficiently large $n$. Taking into account $f(0)=f^{\prime}(0)-1=0$ and the univalence of $f$, we get

$$
\begin{aligned}
(n+p-r) f\left(\frac{1}{n}\right)+f\left(\frac{r}{n}\right) & \neq 0, P_{n, p}^{r}(f ; 0)=\frac{f(0)}{(n+p-r) f\left(\frac{1}{n}\right)+f\left(\frac{r}{n}\right)}=0, \\
\left(P_{n, p}^{r}\right)^{\prime}(f ; 0) & =\frac{\left(L_{n, p}^{r}\right)^{\prime}(f ; 0)}{(n+p-r) f\left(\frac{1}{n}\right)+f\left(\frac{r}{n}\right)}=1,
\end{aligned}
$$


and

$$
\begin{aligned}
(n+p-r) f\left(\frac{1}{n}\right)+f\left(\frac{r}{n}\right) & =\frac{f\left(\frac{1}{n}\right)-f(0)}{\frac{1}{n}} \frac{(n+p-r)}{n}+\frac{f\left(\frac{r}{n}\right)-f(0)}{\frac{r}{n}} \frac{r}{n} \rightarrow f^{\prime}(0) \\
& =1 \text { as } n \rightarrow \infty
\end{aligned}
$$

which means that for $n \rightarrow \infty$, we obtain $P_{n, p}^{r}(f ; z) \rightarrow f(z),\left(P_{n, p}^{r}\right)^{\prime}(f ; z) \rightarrow f^{\prime}(z)$ and $\left(P_{n, p}^{r}\right)^{\prime \prime}(f ; z) \rightarrow f^{\prime \prime}(z)$ uniformly in $\overline{D_{1}}$.

From the hypothesis we obtain $|f(z)|>0$ for all $z \in \overline{D_{1}}$ with $z \neq 0$, which from the univalence of $f$ in $D_{1}$, implies that we can write $f(z)=z g(z)$, with $g(z) \neq 0$, for all $z \in \overline{D_{1}}$, where $g$ is analytic in $D_{1}$ and continuous in $\overline{D_{1}}$.

Writing $P_{n, p}^{r}(f ; z)$ in the form $P_{n, p}^{r}(f ; z)=z Q_{n, p}^{r}(f ; z)$, obviously $Q_{n, p}^{r}(f ; z)$ is a polynomial of degree $\leq n+p-r-1$. Let $|z|=1$. Then we get

$$
\left|f(z)-P_{n, p}^{r}(f ; z)\right|=|z|\left|g(z)-Q_{n, p}^{r}(f ; z)\right|=\left|g(z)-Q_{n, p}^{r}(f ; z)\right|,
$$

which by the uniform convergence in $\overline{D_{1}}$ of $P_{n, p}^{r}(f)$ to $f$ and the maximum modulus principle, gives the uniform convergence in $\overline{D_{1}}$ of $Q_{n, p}^{r}(f ; z)$ to $g(z)$.

Because $g$ is continuous in $\overline{D_{1}}$ and $|g(z)|>0$ for all $z \in \overline{D_{1}}$, there exists an index $n_{1} \in \mathbb{N}$ and $a>0$ depending on $g$, such that $\left|Q_{n, p}^{r}(f ; z)\right|>a>0$, for all $z \in \overline{D_{1}}$ and all $n \geq n_{0}$. Also, for all $|z|=1$, we obtain

$$
\begin{aligned}
\left|f^{\prime}(z)-\left(P_{n, p}^{r}\right)^{\prime}(f ; z)\right| & =\left|z\left[g^{\prime}(z)-\left(Q_{n, p}^{r}\right)^{\prime}(f ; z)\right]+\left[g(z)-Q_{n, p}^{r}(f ; z)\right]\right| \\
& \geq|| g^{\prime}(z)-\left(Q_{n, p}^{r}\right)^{\prime}(f ; z)|-| g(z)-Q_{n, p}^{r}(f ; z)||
\end{aligned}
$$

which gives the uniform convergence of $\left(Q_{n, p}^{r}\right)^{\prime}(f)$ to $g^{\prime}$ by the uniform convergence of $\left(P_{n, p}^{r}\right)^{\prime}(f)$ to $f^{\prime}$ and of $Q_{n, p}^{r}(f)$ to $g$, with the help of the maximum modulus principle. So, for $|z|=1$, we have

$$
\begin{aligned}
\frac{z\left(P_{n, p}^{r}\right)^{\prime}(f ; z)}{P_{n, p}^{r}(f ; z)} & =\frac{z\left[z\left(Q_{n, p}^{r}\right)^{\prime}(f ; z)+Q_{n, p}^{r}(f ; z)\right]}{z Q_{n, p}^{r}(f ; z)} \\
& =\frac{z\left(Q_{n, p}^{r}\right)^{\prime}(f ; z)+Q_{n, p}^{r}(f ; z)}{Q_{n, p}^{r}(f ; z)} \rightarrow \frac{z g^{\prime}(z)+g(z)}{g(z)}=\frac{f^{\prime}(z)}{g(z)}=\frac{z f^{\prime}(z)}{f(z)},
\end{aligned}
$$

which again by the maximum modulus principle, follows

$$
\frac{z\left(P_{n, p}^{r}\right)^{\prime}(f ; z)}{P_{n, p}^{r}(f ; z)} \rightarrow \frac{z f^{\prime}(z)}{f(z)} \text { as } n \rightarrow \infty \text {, uniformly in } \overline{D_{1}} \text {. }
$$

Since $\operatorname{Re}\left(\frac{z f^{\prime}(z)}{f(z)}\right)$ is continuous in $\overline{D_{1}}$, there exists $\xi \in(0,1)$ such that

$$
\operatorname{Re}\left(\frac{z f^{\prime}(z)}{f(z)}\right) \geq \xi, \text { for all } z \in \overline{D_{1}} \text {. }
$$

Thus,

$$
\operatorname{Re}\left(\frac{z\left(P_{n, p}^{r}\right)^{\prime}(f ; z)}{P_{n, p}^{r}(f ; z)}\right) \rightarrow \operatorname{Re}\left(\frac{z f^{\prime}(z)}{f(z)}\right) \geq \xi>0
$$

uniformly on $\overline{D_{1}}$, i.e. for any $0<\mu<\xi$, there is $n_{0}$ such that for all $n \geq n_{0}$, we get

$$
\operatorname{Re}\left(\frac{z\left(P_{n, p}^{r}\right)^{\prime}(f ; z)}{P_{n, p}^{r}(f ; z)}\right)>\mu>0, \text { for all } z \in \overline{D_{1}} \text {. }
$$


Since $P_{n, p}^{r}(f ; z)$ differs from $L_{n, p}^{r}(f ; z)$ only by a constant, this proves the starlikeness of $L_{n, p}^{r}(f ; z)$.

The proofs in the cases when $f$ is convex or spirallike of order $\gamma$ are similar and follow from the following uniform convergences (on $\overline{D_{1}}$ or on $\overline{D_{\rho}}$ with $0<\rho<1$ ) as $n \rightarrow \infty$

$\operatorname{Re}\left(\frac{z\left(P_{n, p}^{r}\right)^{\prime \prime}(f ; z)}{\left(P_{n, p}^{r}\right)^{\prime}(f ; z)}\right)+1 \rightarrow \operatorname{Re}\left(\frac{z f^{\prime \prime}(z)}{f^{\prime}(z)}\right)+1$ and $\operatorname{Re}\left(e^{i \gamma} \frac{z\left(P_{n, p}^{r}\right)^{\prime}(f ; z)}{P_{n, p}^{r}(f ; z)}\right) \rightarrow \operatorname{Re}\left(e^{i \gamma} \frac{z f^{\prime}(z)}{f(z)}\right)$.

\section{REFERENCES}

[1] Anastassiou, G. A. and Gal, S. G., Approximation by complex Bernstein-Schurer and Kantorovich-Schurer polynomials in compact disks , Comput. Math. Appl., 58 (2009), 734-743

[2] Bernstein, S. N., Démonstration du théorème de Weierstrass fondée sur le calcul des probabilités, Commun. Kharkov Math. Soc., 13 (1912/1913), 1-2

[3] Bustamante, J. and Quesada, J. M., A property of Ditzian-Totik second order moduli , Appl. Math. Lett., 23 (2010), No. 5, 576-580

[4] Çetin, N. and Başcanbaz-Tunca, G., Approximation by a new complex generalized Bernstein operators, An. Univ. Oradea Fasc. Mat., Tom XXVI (2019), No. 2, 127-139

[5] DeVore, R., Lorentz, G. G., Constructive Approximation, Springer, Berlin, 1993

[6] Gal, S. G., Approximation by Complex Bernstein and Convolution Type Operators, Series on Concrete and Applicable Mathematics, vol. 8, World Scientific Publishing Co. Pte. Ltd., Hackensack, NJ, 2009

[7] Graham, I. and Kohr, G., Geometric Function Theory in One and Higher Dimensions, Pure and Applied Mathematics, Marcel Dekker, 255, New York, 2003

[8] Jiang, B. and Yu, D., On approximation by Stancu type Bernstein-Schurer polynomials in compact disks, Results Math., 72 (2017), 1623-1638

[9] Schurer, F., Linear positive operators in approximation theory, Math. Inst. Techn. Univ. Delft Report, 1962

[10] Stancu, D. D., Quadrature formulas constructed by using certain linear positive operators, Numerical Integration (Proc. Conf., Oberwolfach, 1981), ISNM 57 (1982) 241-251, Birkhäuser Verlag, Basel

[11] Stancu, D. D., Approximation of functions by means of a new generalized Bernstein operator, Calcolo, 20 (1983), No. 2, 211-229

[12] Yang, R., Xiong, J. and Cao, F., Multivariate Stancu operators defined on a simplex, Appl. Math. Comput., 138 (2003), 189-198

DEPARTMENT OF MATHEMATICS

ANKARA HACI BAYRAM VELI UNIVERSITY

POLATLi FACULTY OF SCIENCE AND LETTERS

06900, ANKARA, TURKEY

Email address: nursel cetin07@gmail. com 